\title{
On Deflection as a Performance Criterion in Detection
}

Bernard Picinbono, Fellow, IEEE

\section{The pdf copy of the final published text can be obtained from the author at the folowing address : bernard.picinbono@1ss.supelec.fr}

Reference of this text : IEEE Trans. on Aerospace and Electronic Systems, Vol. 31, T. 3, pp. $1072-1081,1995$

\begin{abstract}
The performance of detection system is usually characterized by the receiver operating characteristics (ROC) curves. Because of the complexity of the calculation of such curves simpler performance criteria are useful. Among these, the deflection criterion, or output signal-to-noise ratio, is one of the most interesting. The advantages and limitations of the deflection are first analyzed. Attention is especially paid to the relation between singular detection and Sinite deflection Maximization of the deflection in the class of linear-quadratic (L-Q) system is also discussed and it is shown that in order to detect a deterministic signal in some kinds of non-Gaussian noise a quadratic term can introduce an almost singular detection This situation is analyzed by computer simulations and comparisons between results obtained via the deflection or the ROC curves indicate the interest of the deflection in the analysis of the performance of some nonlinear system.
\end{abstract}

\section{Manuscript received}

B. P. is with the Laboratoire des Signaux et Systèmes (L2S), a joint laboratory of the C.N.R.S. and the École Supérieure d'Électricité, Plateau de Moulon, 3 rue Joliot-Curie 91192, Gif sur Yvette, France. The L2S is associated with the University of Paris-Sud, France. E-mail: bernard.picinbono@1ss.supelec.fr, . 


\section{INTRODUCTION}

The basic detection problem can be stated as a hypothesis testing problem. In the simplest case of radar or sonar it can be reduced to the choice between the hypothesis $H_{0}$, or noise only, and the hypothesis $H_{1}$, or signal present in noise. In the case where these hypotheses are simple, the optimum receiver must compare the likelihood ratio (LR) to a threshold and the performance is characterized by the receiver operating characteristics curves (ROC) giving the detection probability $\mathrm{p}$ in terms of the false alarm probability $\alpha[1,21$. It is sometimes said that the LR receiver is the optimum receiver and this is related to the fact that the LR is a sufficient statistic for the choice between $H_{0}$ and $H_{1}$. In reality there is not only one optimum receiver but a family because any strictly monotone function of the LR exhibits the same ROC curve. It is therefore better to talk about an optimum class of equivalence containing all the receivers equivalent to the $\mathrm{LR}$ in terms of performance.

Unfortunately in many practical problems it is impossible to implement the LR receiver either because of its complexity or because the probability densities of the observation under $H_{0}$ or $H_{1}$ are not completely known. This appears especially in the case of signal detection in non-Gaussian noise [3].

Furthermore in order to compare various detection procedures it is a rather formidable task to calculate the ROC curves, so other criteria of comparison must be used (see [3, p. 461). Among several possible criteria, the deflection criterion (or output signal-to-noise ratio) appears interesting. It has been used for a long time and can be justified by various arguments.

However it remains an open question to know whether an increase of deflection will give an increase of performance in terms of ROC. Even though there is no general answer to this question, the purpose here is to introduce a partial answer in some specific situations. In particular it is interesting to verify whether receivers introducing infinite value of deflection leads to singular detection, which means a zero false alarm probability and a detection probability equal to one.

The paper is organized as follows. In the second section we analyze the advantages and limitations of the deflection criterion. In the third section we describe a statistical situation corresponding to a physical problem where the LR, although known, cannot be implemented, and where singular detection can be achieved by using a linear-quadratic (L-Q) receiver. In the last section the ROC curves of linear and some nonlinear receivers are calculated and the advantage of taking into account, for example, a quadratic term, even for the detection of a deterministic signal, clearly appears. 


\section{DeFLECTION CRITERION}

Let $\mathbf{x}$ be a real observation vector and $(\mathbf{x})$ the output of an arbitrary receiver. The deflection associated with $(\mathbf{x})$ is defined by

$$
D(S)=\frac{\left[E_{1}(S)-E_{0}(S)\right]^{2}}{V_{0}(S)},
$$

where $E_{0}$ and $E_{1}$ mean expectations under $H_{0}$ and $H_{1}$, respectively, $V_{0}$ means the variance under $H_{0}$. It is of course assumed that $S(\mathbf{x})$ is such that all these quantities are finite. The deflection is sometimes called the output signal-to-noise ratio (see [3, p. 531). The deflection has a long history, predating the LR theory and maximization of deflection was considered under various assumptions in [4' - 8]] It is worth pointing out that the use of deflection criterion leads to the LR receiver in many cases of practical importance. Consider first the detection of a deterministic signal in a Gaussian noise. The LR receiver is then linear and often called the matched filter [2]. The same filter is obtained by maximizing the deflection $D(S)$ for systems $S(x)$ linear with respect to the observation x. Similarly, the LR receiver associated to the detection of a Gaussian signal in Gaussian noise is quadratic with respect to $\mathbf{x}$. Its structure is the same as that obtained by maximizing the deflection of quadratic systems $\mathbf{x}$. This was obtained in terms of square law detector and linear filtering in [4] and generalized for quadratic filters in [5]. In conclusion, when Gaussian assumption is introduced, the maximisation of the deflection leads to the same structures as LR theory. This is a good reason to study in detail the advantages and disadvantages of deflection in more general situations.

\section{A. Advantages of the Deflection}

Let us summarize the various advantages of the deflection in detection problems.

1) By using the deflection criterion the system $S(\mathbf{x})$ is characterized by only one number instead of by a curve. It is then in general much easier to calculate.

2) There is a relation between the LR receiver and the optimum receiver in terms of deflection analyzed in [8]. Let us summarize the main results. At first the system $S(\mathbf{x})$ maximizing (1) is none other than the LR $L(\mathbf{x})$, ratio of the probability densities functions of $\mathbf{x}$ under $H_{0}$ and $H_{1}$, respectively. Furthermore, if there are some constraints on $S(\mathbf{x})$, the receiver maximizing the deflection under these constraints is the receiver the output of which is the mean square estimation of $L(\mathbf{x})$. As minimizing the mean square error can be interpreted as minimizing a distance, this result means that the optimum detector in terms of deflection is the receiver such that its distance to the LR is minimum. In this perspective the output of the matched filter that maximizes $D(S)$ in the class of linear systems is the best linear approximation in the mean square sense of the LR. 
3) The deflection criterion is well adapted to the context of "higher-order statistics" widely used recently in many areas of signal processing [7-10]. As an example suppose that we want to test Ho against HI with an incomplete knowledge about the statistics under these two hypotheses. Let us assume that this knowledge is limited to some moments, without any other information on the structure of the probability distributions.

Let us begin with a knowledge limited to the second order. This means that we know the mean values $\mathbf{m}_{0}$ and $\mathbf{m}_{1}$ of $\mathbf{x}$ under $H_{0}$ and $H_{1}$, respectively, and also the covariance matrice $\boldsymbol{\Gamma}_{0}$ of $\mathbf{x}$. From this knowledge it is possible to find the linear receiver $S(\mathbf{x})$ maximizing the deflection. The assumption of linearity means that $S(\mathbf{x})=\mathbf{h}^{T} \mathbf{x}$ and the output mean value is $\mathbf{h}^{T} \mathbf{x}$ then $\mathbf{h}^{T} \mathbf{m}$ where $\mathbf{m}$ is the expectation value of $\mathbf{x}$. As a result the numerator of (1) is $\mathbf{h}^{T}\left(\mathbf{m}_{1}-\mathbf{m}_{0}\right)$. Similarly, by using the fact that the variance of $\mathbf{h}^{T} \mathbf{x}$ is $\mathbf{h}^{T} \boldsymbol{\Gamma}_{0} \mathbf{h}$, we deduce that the deflection of $S(\mathbf{x})$ is

$$
D(S)=\frac{\left[\mathbf{h}^{T}\left(\mathbf{m}_{1}-\mathbf{m}_{0}\right)\right]^{2}}{\mathbf{h}^{T} \boldsymbol{\Gamma}_{0} \mathbf{h}} .
$$

Suppose now that $\Gamma_{0}$ has no zero eigenvalue, which means that it is positive definite. In this case, as indicated in [9, p. 1441, it is possible to consider that $\mathbf{a}^{T} \boldsymbol{\Gamma}_{0} \mathbf{b}$ is a scalar product between two vectors $\mathbf{a}$ and $\mathbf{b}$. The Schwarz inequality yields the maximaum value of $D(S)$ by

$$
S_{\text {opt }}(\mathbf{x})=\left(\mathbf{m}_{1}-\mathbf{m}_{0}\right)^{T} \boldsymbol{\Gamma}_{0}^{-1} \mathbf{x}
$$

which is the classical matched filter. Of course any linear system proportional to (3) yields the same deflection. In conclusion if our knowledge about the two hypotheses is limited to the second order, which means that only $\mathbf{m}_{0}, \mathbf{m}_{1}$ and $\boldsymbol{\Gamma}_{0}$ are known, it is possible to calculate the linear system maximizing the deflection and this system is the classical matched filter. This gives some significance to the deflection criterion.

If our knowledge is limited to the fourth-order moments of $\mathbf{x}$ under $H_{0}$ and $H_{1}$, it is possible to use an L-Q system in order to maximize the deflection. Various properties of such systems are analyzed in [7] and extensions to higher order properties are given in [8 and 101.

As an example, it follows from the previous studies that in order to detect a deterministic signal in a noise for which only some moments are known, the linear receiver is not necessarily the best one, as it is in the case where the noise is Gaussian. The use of nonlinear terms can significantly increase the deflection and there are some cases, analyzed below, where the introduction of a quadratic term can generate an infinite deflection, or a singular detection, that would be impossible to achieve with only a linear system. However one may ask whether this increase of the deflection is related to the performance calculated in terms of ROC curves. It is of course impossible to answer this question, because in the 
framework in which the problem is stated, the ROC curves cannot be calculated. In fact the knowledge of only a limited number of moments is insufficient to obtain the probability distributions, the knowledge of which is necessary to calculate the ROC curves. This question is analyzed below in another context.

4) There is finally a last argument in favor of the deflection. It concerns the asymptotic case (see [3, p. 471). If the components of $\mathrm{x}$ are independent and identically distributed (IID) random variables it is possible to use a generalized correlator detector (GCD) (see [3, p. 371) and, by invoking the Central Limit Theorem, to assume that the output is Gaussian. Then it becomes again possible to calculate the ROC curve and it appears that maximizing the deflection becomes equivalent to optimizing the performances calculated in terms of ROC.

\section{B. Limits of the Deflection}

Except for the asymptotic case indicated just above, the deflection criterion has some limitations with respect to its use in detection problems.

1) The physical meaning of the deflection is not obvious. In fact, after the calculation of the system giving the maximum of the deflection, the question becomes: how to use this system in a detection procedure? As the problem is to decide between two possible hypotheses we can compare the output of the optimum system with a threshold to make the decision. However, this rule has no relation with the deflection, and we see below that other procedures must sometimes be applied.

2) Let $S_{1}$ and $S_{2}$ be two systems and suppose that their deflections satisfy $D\left(S_{1}\right)<D\left(S_{2}\right)$. It is then impossible to prove generally that for a given false alarm probability $\alpha$, the detection probability of $S_{2}$ is greater than that of $S_{1}$. This can be true for some values of $\alpha$ and untrue for other ones. In other words a greater deflection does not necessarily give a better performance in terms of ROC curves.

This does not mean that the deflection criterion must be completely rejected. It must be used with some care, and let us remind that, as noticed previously, it is sometimes the only performance criterion that can be used.

\section{LINEAR-QUADRATIC GENERALIZED CORRELATOR DETECTORS}

Suppose now that the components $x_{i}$ of the observation vector are IID random variables under $H_{0}$ and $H_{1}$. In this case the LR receiver can be realized with the structure of a GCD.

In fact the $\log \operatorname{LR} \Lambda(\mathbf{x})$, equivalent in terms of detection to the LR itself, is equal to

$$
\Lambda(\mathbf{x})=\sum_{i=0}^{n} \lambda\left(x_{i}\right)
$$

where $\lambda(x)$ is the logarithm of $p_{1}\left(x / p_{0}(x)\right)$, LR common to each component $x_{i}$ of $\mathbf{x}$. 
Suppose now that, either because of the complexity of this LR or because only the first moments of $x$ are known, we approximate this receiver by replacing $\lambda(x)$ by a function $g(x)$ which is L-Q in $x$. The problemx is then to calculate $y=g(x)$ in order to maximize the deflection. The output of the system can then be written as

$$
S(\mathbf{x})=\sum_{i=0}^{n} g\left(x_{i}\right) .
$$

It is obvious that $E_{i}(S)=n E_{i}[(g(x))]$, where $i=0$ or $i=1$. Furthermore, as the quantities $g\left(x_{i}\right)$ are also IID random variables, $V_{0}(S)=n V_{0}(g)$. As a consequence the deflection associated with $S$ is easily deduced from the one obtained with $g$, and thus we only have to calculate this deflection.

For this we assume that the moments

$$
m_{k}=E_{0}\left(x^{k}\right)
$$

are known for $1 \leq k \leq 4$ and also that $m_{1}=0$. Similarly we suppose that the moments

$$
\mu_{k}=E_{1}\left(x^{k}\right)
$$

are known for $1 \leq k \leq 2$. As the deflection of $g($.) remains unchanged if we replace $g$ by $\lambda g+\mu$, where $\lambda$ and $\mu$ are arbitrary constants, we can impose that $E_{0}(y)=0$, and the structure of the most general L-Q system is

$$
y=a x+b\left(x^{2}-m_{2}\right) .
$$

The interest of assuming that $E_{0}(y)=0$ is only to simplify the calculation of the variance of $y$ that becomes simply $E_{0}\left(y^{2}\right)$. The problem is now to find $a$ and $b$ in such a way that the deflection $D(y)$ is maximum. For this calculation it is appropriate to write $y$ in the form

$$
y=\mathbf{H}^{T} \mathbf{X}
$$

where $H$ and $\mathbf{X}$ are vectors $\mathbb{R}^{2}$ defined by

$$
\mathbf{H}^{T}=[a, b] ; \mathbf{X}^{T}=\left[x, x^{2}-m_{2}\right] .
$$

It is clear that $E_{0}(\mathbf{X})=\mathbf{0}$ and that

$$
E_{1}\left(\mathbf{X}^{T}\right)=\left[\mu_{1}, \mu_{2}-m^{2}\right] \triangleq \mathbf{S}^{T} \triangleq[s, \gamma]
$$

Similarly the covariance matrix $\mathbf{R}$ of $\mathbf{X}$ is

$$
\mathbf{R}=\left(\begin{array}{cc}
m_{2} & m_{3} \\
m_{3} & m_{4}-m_{2}^{2}
\end{array}\right)
$$

With these notations the deflection of $y$ can be expressed as

$$
D(y)=\frac{\left(\mathbf{H}^{T} \mathbf{S}\right)^{2}}{\mathbf{H}^{T} \mathbf{R H}} .
$$


This expression has the same structure as (2) and the same procedure can be used for its maximization. As a result the maximum valuc of $D$ is given by an expression similar to the last term of (3.a) and now equal to

$$
D_{\max }=\mathbf{S}^{Y} \mathbf{R}^{-1} \mathbf{S}
$$

This maximum value is reached if $\mathbf{H}$ is a solution of the equation

$$
\mathbf{R H}=\mathbf{S} .
$$

Note that any vector proportional to this solution yields the same deflection (13).

By using the value of $S$ given by (11) we can write the linear equation (15) in the form

$$
\begin{gathered}
m_{2} a+m_{3} b=s \\
m_{3} a+\left(m_{4}-m_{2}^{2}\right) b=\gamma
\end{gathered}
$$

The solution of this system of equations is straightforward and the results are

$$
\begin{gathered}
a=\Delta^{-1}\left[\left(m_{4}-m_{2}^{2}\right) s-m_{3} \gamma\right] \\
b=\Delta^{-1}\left[-m_{3} s+m_{2} \gamma\right] \\
\Delta=m_{2}\left(m_{4}-m_{2}^{2}\right)-m_{3}^{2} \\
D_{\max }=s^{2} m_{2}^{-1}+\Delta^{-1}\left(m_{2} \gamma-m_{3} s\right) m_{2}^{-1} .
\end{gathered}
$$

Let us finally discuss the case of the detection of a deterministic signal. Under $H_{0}$ we have $x=n$ (noise only) and under $H_{1}$ we have $x=n+s$, where $s$ is deterministic. By calculating $\mu_{2}$ we deduce from (11) that $\gamma=s^{2}$. As a result we see that the optimum L-Q system is purely linear only if $s=m_{3} / m_{2}$.

To be complete we must now discuss the case where $\Delta=0$. This relation means that $\mathbf{R}$ is now a rank 1 matrix. The deflection can then become infinite if the projection of the signal $\mathbf{S}$ onto the vector $\mathbf{u}_{0}$, one of the eigenvectors of $\mathbf{R}$ with zero eigenvalue, is non-zero. The vector $\mathbf{u}_{0}$ defined by

$$
\mathbf{u}_{0}=\left[m_{3},-m_{2}\right]^{T}
$$

is eigenvector of $\mathbf{R}$ with zero eigenvalue when $\Delta=0$. Therefore, there is singular detection if

$$
\mathbf{S}^{T} \mathbf{u}_{0}=m_{3} s-m_{2} \gamma \neq 0
$$

The corresponding L-Q system deduced from (22) is

$$
y=k\left[m_{3} x-m_{2}\left(x^{2}-m_{2}\right)\right]
$$


where $\mathrm{k}$ is an arbitrary constant. In this case the deflection indeed becomes infinite because the numerator of (13) is finite and the denominator is zero.

Let us present a physical interpretation of this situation. When $\Delta=0$, the variance of $y$ is zero. This means that under $H_{0}, y(x)$ has a zero mean value and a zero variance, which implies that $y(x)=0$ with probability one. On the other hand if $m_{2} \gamma-m_{3} s \neq 0$, then $E_{1}(y) \neq 0$. This explains why the deflection is infinite.

Let us now analyze more precisely the relation between this situation and the singular detection. Let $p$ be the probability that $y(x)=0$ under $H_{1}$. Suppose that the decision rule is simply

$$
y(x)=0 \rightarrow H_{0} \quad: \quad y(x) \neq 0 \rightarrow H_{1}
$$

It is clear that the false alarm probability $\alpha$ is zero. On the other hand the detection probability $\beta$ is $1-p$, and this means that even if the deflection is infinite, there is singular detection only if $p=0$. This is especially the case when $y$ is a continuous random variable under $H_{1}$. However, we must not ignore the case of discrete random variables used in the following discussion.

In the case where we have no a priori information about the sign of $y$ it is more appropriate to replace the decision rule (25) by

$$
|y(x)|=0 \rightarrow H_{0} \quad: \quad|y(x)|>0 \rightarrow H_{1}
$$

which makes use of the function $|y(x)|$. This shows that, even if the function $y(x)$ giving an infinite deflection is perfectly determined, its use in a decision procedure is somehow arbitrary. This is due to the fact, already indicated, that the deflection itself does not introduce a decision rule.

However, the possible singular detection that is characterized in terms of ROC is mainly due to the fact that $y(x)$ given by (24) is an L-Q system. For the same a priori knowledge of the moments, a linear system only would not lead to singular detection. In other words, taking into account a quadratic term can yield a strong change in the behavior of a detection system. This point is analyzed in more detail in the next section.

Let us now invzstigate the consequences of the condition $\Delta=0$ on the structure of the random variable $x$ under $H_{0}$. This condition implies, as seen before, that the covariance matrix $\mathbf{R}$ of the vector $\mathbf{X}$ has one zero eigenvalue, which means that $\mathbf{R}$ is a rank one matrix. Consequently, the two components of $\mathbf{X}$ defined by (10) are proportional with probability one, which yields

$$
x^{2}-m^{2}=c x .
$$

Consequently, the random variable $x$ can take only two values $x_{1}$ and $x_{2}$, the solutions of this equation. 


\section{EXAMPLES}

Signals taking only two values are very common in communication problems. The best known example is probably the random telegraph signal (see $[9$, p. 3341) taking only the values \pm 1 , the changes appearing at the time instants of a Poisson process.

Suppose now that $x$ is a zero-mean random variable taking only the values $x_{1}$ and $x_{2}$ with the probabilities $p$ and $1-p$, respectively. The assumption of zero mean value implies that $x_{2}=-p x_{1}(1-p)^{-1}$ The moments $m_{2}, m_{3}$, and $m_{4}$ are readily calculated and by inserting their values in (20) we verify that $\Delta$ given by (20) is zero. Under $H_{0}$ we have $\left(x-x_{1}\right)\left(x-x_{2}\right)=0$ and thus the function

$$
y(x)=x^{2}-\left(x_{1}+x_{2}\right) x+x_{1} x_{2}
$$

will generate singular detection if the conditions given in the previous section are fulfilled.

It is clear from (28) that, as indicated above, singular detection is only achievable if a quadratic term is introduced in the L-Q system. Suppose now that $p=1 / 2$. In this case $x_{1}=-x_{2}$ and if $x_{1}=1$ we deduce that the system (28) can be written as

$$
y(x)=x^{2}-1
$$

It is exactly the situation obtained, for example, with the random telegraph signal, in which case $y(x)=0$ under $H_{0}$.

We now analyze this situation in more depth and in order to simplify the terminology we introduce the term of binary noise for a signal taking only two values, like the random telegraph signal.

Suppose that the noise is also a binary signal. This situation can arise when the noise comes from the interference due to a binary signal used in another channel. Thus this binary signal is a useful signal for one receiver and an interference, or a noise, for the other receiver.

We always use the system described above and will only describe the situation before the accumulator. In other words, we want to study the use of only one random variable $x_{i}$ for the purpose of detection.

Under $H_{0}$ the observation $\mathrm{x}$ takes only the values \pm 1 with the probabilities $1 / 2$. As the signal is deterministic, the observation under $H_{1}$ takes the values \pm 1 with the same probabilities. As there are no probability density functions, there is no simple expression for the LR and our purpose is to compare the linear receiver $y_{L}(x)=x$ and the optimum L-Q receiver given by (29) or $y_{L Q}(x)=x^{2}-1$. Note that in this specific example the optimum L-Q receiver is purely quadratic.

As the observation under $H_{0}$ and $H_{1}$ is a discrete random variable, the ROC curves are limited to points. In order to obtain a continuous curve the standard procedure is to use a randomized test (see [2, p. 431) which leads to join the points by straight lines. The decision is obtained by comparison with a threshold and $H_{1}$ is decided if the decision function is greater than this threshold. 
We then want to compare the three following detection procedures

$$
\begin{gathered}
y_{L}(x)=x \gtrless t \\
y_{L Q}(x)=x^{2}-1 \gtrless t \\
\left|y_{L Q}(x)\right|=\left|x^{2}-1\right| \gtrless t
\end{gathered}
$$

for a small value of $s$. In this problem this means $s<2$, which ensures that $y_{L Q}(x)$ takes negative values in order to obtain a difference between $y_{L Q}(x)$ and $\left|y_{L Q}(x)\right|$.

The ROC curve of the linear receiver shows that for a zero false alarm probability the detection probability can be non-zero but its maximum value is equal to 0.5. This is not exactly a situation of singular detection. Furthermore the point $\alpha=\beta=0.5$ belongs to the ROC curve. This exhibits a very poor behavior because for $\alpha=0.5$ there is no difference between the situations signal present or signal absent.

The ROC curve of the L-Q receiver (31) shows that this receiver must be rejected because it can give a detection probability smaller than the false alarm probability.

Finally, the behavior of the receiver using $\left|y_{L Q}(x)\right|$ shows the property of singular detection. As a matter of fact for $0<t<s^{2}-2 s$ we have $\alpha=P\left[\left|y_{L Q}\right|>t \mid H_{0}\right]=0$ and $\beta=P\left[\left|y_{L Q}\right|>t \mid H_{1}\right]=1$, which means singular detection.

From these remarks we can deduce two main features. First, as indicated previously, detection performance can be greatly improved by the inclusion of a quadratic term, even for a deterministic signal. Secondly, the final decision rule cannot be deduced from the calculations giving the maximum deflection. This calculation gives the L-Q function $x^{2}-1$. However this is not the function that must be used for the comparison with a threshold.

\section{CONClusion}

We have introduced the deflection criterion in signal detection problems. This criterion is well adapted to design optimum systems for detection when the statistical properties of the signal and the noise are not entirely known. This is especially the case when this knowledge is limited to moments up to a given order. If this order is two, the optimum system in terms of deflection is the classical matched filter. If this order is four, it is possible to design optimum L-Q receivers for detection and more generally if this order is $2 n$ the deflection criterion allows us the calculation of an optimum polynomial system of order $n$. There is no formal relationship between optimum systems in terms of deflection and in terms of performance evaluated with ROC curves. However we have shown with some examples that the tendency 
towards singular detection can be evaluated both with ROC curves and with deflection criteria. These examples also show that there are some statistical situations where the use of L-Q systems instead of only linear ones can significantly improve the detection performance with respect to the use of only linear systems even in the case of deterministic signals. This improved performance is easily obtained in terms of deflection but appears also on the ROC curves calculated for some specific statistical distributions. Unfortunately this property has no reason to be general and it would be interesting to investigate more extensively its domain of validity.

\section{REFERENCES}

[1] Helstrom, C. W. (1968)

Statistical Theory of Signal Detection (2nd ed.).

Oxford: Pergamon Press, 1968.

[2] Van Trees , H. L. (1968)

Detection, Estimation, and Modulation Theory, Part I.

New York: Wiley, 1968.

[3] Kassam, S. A. (1988)

Signal Detection on Non-Gaussian Noise.

New York Springer-Verlag, 1988.

[4] Eckardt, C. (1952)

Optimal rectifier systems for the detection of steady signals.

Technical report S.10, ref. 52 11, Scripps Institute of Oceanography, University of California, Mar. 1952.

[5] Baker, C. R. (1966) Optimum quadratic detection of a random vector in Gaussian noise. IEEE Trans. Trans. Commun., COM-14 (Dec. 1966), 80Z-805.

[6] Gardner, W. A. (1976) Structurally constrained receivers for signal detection and estimation. IEEE Trans. Trans. Commun., COM-24 (1976), 578-592.

[7] Picinbono, B., and Duvaut, P. (1988) Optimal linear-quadratic systems for detection and estimation. IEEE Transactions on Information Theory, 34 (Mar. 1988), 304-311.

[8] Picinbono, B., and Duvaut, P. (1990) Geometrical properties of optimal Volterra filters for signal detection. IEEE Transactions on Information Theory, 36 (Mar. 1990), 1061-1068.

[9] Picinbono, B. (1993)

Random Signals and Systems.

Englewood Cliffs, NJ: Prentice-Hall, 1993.

[10] Picinbono, B., and Duvaut, P. (1990) Le filtrage de Volterra transverse et complexe en traitement du signal. IEEE Traitement du signal, 7 (1990), 451-476. 